\title{
Effect of aloe polysaccharide on caspase-3 expression following cerebral ischemia and reperfusion injury in rats
}

\author{
ZHONG-QIAN LU ${ }^{1}$, YI-JUN DENG ${ }^{1}$ and JIAN-XIA LU ${ }^{2}$ \\ ${ }^{1}$ The Intensive Care Unit, The First People's Hospital of Yancheng City, Yancheng 224005; \\ ${ }^{2}$ Department of Medical Technology, Yancheng Health Vocational and Technical College, \\ Yancheng, Jiangsu 224006, P.R. China
}

Received February 4, 2012; Accepted April 24, 2012

DOI: $10.3892 / \mathrm{mmr} .2012 .927$

\begin{abstract}
Stroke is a leading cause of cardiovascular morbidity, economic and social burden and mortality. Novel approaches are needed to address stroke prevention and treatment. The purpose of this study was to explore the effects of aloe polysaccharide on caspase-3 expression following cerebral ischemia reperfusion injury in rats. Male Wister rats were randomly divided into 5 groups (16 rats in each group): aloe polysaccharide, ginkgo leaf tablet, nimodipine, model and sham surgery groups. The rats were administered the appropriate drug or normal saline for 7 days by gavage. A rat model of cerebral ischemia and reperfusion injury was established using the middle cerebral artery occlusion (MCAO) model. Caspase-3 protein and mRNA expression levels in the cerebral cortex were detected by immunohistochemistry and reverse transcription-polymerase chain reaction (RT-PCR), respectively. Results showed that caspase-3 protein and mRNA expression levels in the cerebral cortex in the aloe polysaccharide, ginkgo leaf tablet and nimodipine groups were significantly lower compared with the model group and were higher than the sham surgery group $(\mathrm{P}<0.05)$. No significant difference was observed in caspase-3 protein and mRNA expression among the aloe polysaccharide, the ginkgo leaf tablet and the nimodipine groups ( $\mathrm{P}>0.05)$. In conclusion, aloe polysaccharide has a protective effect on cerebral ischemia that may be due to the inhibition of neuronal cell apoptosis.
\end{abstract}

\section{Introduction}

Cerebrovascular disease, also known as stroke, is a clinically common disease characterized by high morbidity, mortality,

Correspondence to: Professor Yi-Jun Deng, The Intensive Care Unit, The First People's Hospital of Yancheng City, No. 16 Yuehe Road, Tinghu District, Yancheng, Jiangsu 224005, P.R. China

E-mail: dengyj2003@163.com

Key words: aloe polysaccharide, cerebral ischemia, reperfusion injury, cell apoptosis, caspase-3 disability and recurrence. Ischemic cerebrovascular disease (cerebral infarction) accounts for more than $70 \%$ of cerebrovascular disease. Two million individuals in China develop cerebrovascular disease each year, with an incidence rate of 120/100,000 individuals. Cerebrovascular disease has a detrimental effect on the patients' family and society (1). Therefore, there is an urgent need to understand the pathogenesis of this disease to improve treatment and prevention. Currently, studies concerning stroke pathogenesis mainly involve energy deficit, excitatory amino acid toxicity, cell depolarization, calcium influx, inflammation and apoptosis (2). Of these, neuronal apoptotic mechanisms currently draw the most focus (3). Apoptosis is regulated by various cytokines including the caspase enzyme family (4). Caspase- 3 plays a key role in neuronal apoptosis following cerebral ischemia reperfusion (5). In this study, a rat model of cerebral ischemia reperfusion injury was established to observe the effect of aloe polysaccharides on neuronal caspase- 3 protein and mRNA expression. This study provides mechanistic insight into the effects of aloe polysaccharides on ischemic brain injury.

\section{Materials and methods}

Animals, drugs and reagents. Male Wistar rats (weight,

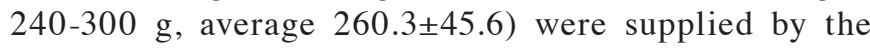
Experimental Animal Center of Jiangsu Province (Nanjing City, China). The rats had free access to food and water, but were fasted for $12 \mathrm{~h}$ prior to surgery. The aloe polysaccharides were prepared by the Department of Pharmacology, Nanjing University of Chinese Medicine (Nanjing, China). Other supplies included nimodipine tablets (Shanghai SCOND Pharmaceutical Co., Ltd., Shanghai, China), Ginkgo biloba tablets (Shanghai Xingling Science and Technology Pharmaceutical Co., Ltd., Shanghai, China), caspase-3 rabbit anti-mouse polyclonal antibody (Santa Cruz Biotechnology, Inc., Santa Cruz, CA, USA), TRIzol reagent and primer nucleotide fragments (Invitrogen, Carlsbad, CA, USA) and RNA PCR Kit (Toyobo Co., Ltd., Osaka, Japan).

Experimental apparatus. The equipment used in the experiments includes an automatic biological tissue hydroextractor (ZT-12P2, Xiaogan Yaguang Medical Electronics Co., Ltd., Hubei, China), a refrigerated investing machine (YB-6LF, 
Xiaogan Yaguang Medical Electronics Co., Ltd), semiautomatic rotary microtome (HM340E, Microm, Walldorf, Germany), an optical microscope (CX31, Olympus, Tokyo, Japan), professional digital camera (DPIXEL200, Guangzhou Tykor Computer Co., Ltd.), Image-Pro Plus 6.0 image analysis software (Media Cybernetics, Bethesda, MD, USA), PCR amplification meter (Type 5331, Eppendorf, Hamburg, Germany); DYY-III Type 613 electrophoresis apparatus, Type DYY-33B horizontal electrophoresis tank (Beijing 61 Factory, Beijing, China) and sterilized workstations for medical use (Suzhou Purification Equipment Corporation, Suzhou, China).

Grouping and administration. Rats $(\mathrm{n}=80)$ were randomly divided into five groups ( $\mathrm{n}=16$ each): aloe polysaccharide $(60 \mathrm{mg} / \mathrm{kg})$, nimodipine treatment $(21.6 \mathrm{mg} / \mathrm{kg})$, Ginkgo biloba $(135 \mathrm{mg} / \mathrm{kg})$, model control and sham surgery groups. The rats were administered an equal volume of saline and received the treatments by gavage continuously for 7 days.

Establishment of model of right middle cerebral artery occlusion. A modified method of that described by Longa et al (6) was used to establish right middle cerebral artery occlusion (MCAO) 30 min after the administration of the final treatment. Following MCAO surgery (30 min after the restoration of consciousness), the neurological deficit of the rats was scored and those with post-MCAO scores of 1-3 were included in the study. The qualified rats from the five groups were then divided into two groups; one group was used to measure caspase-3 protein expression in the cerebral cortex $(n=6$ rats per group) and the other was used to measure caspase- 3 mRNA expression (the remaining rats).

Caspase-3 protein detection. The rats were anesthetized with an intraperitoneal injection of $10 \%$ chloral hydrate $(350 \mathrm{mg} / \mathrm{kg})$ followed by rapid thoracotomy to expose the heart. The right atrial appendage was cut open, a tube was inserted from the left ventricle into the ascending aorta and approximately $150 \mathrm{ml}$ of heparin saline was infused over $5 \mathrm{~min}$. Then, $200 \mathrm{ml}$ of $0.1 \mathrm{~mol} / 1$ of phosphate buffer containing $4 \%$ paraformaldehyde was infused slowly to allow tissue fixation. When the infusion was complete, a cut was made from the foramen magnum to remove the whole brain. The tissue was placed in the same fixative at $4^{\circ} \mathrm{C}$. Paraffin sections were sliced to a thickness of $4 \mu \mathrm{m}$ and the slices were treated using the SP method. When the reaction was terminated, the slices were re-stained with hematoxylin, then rinsed with clear water, stained blue and treated with gradient ethanol dehydration, dimethylbenzene verification, sealed with neutral resins and observed. The caspase-3 immunohistochemistry results were observed under a light microscope. Images of the slides were captured in 10 high-power fields and 100 cells were counted per field for a total of 1,000 cells. The average positive rate (\%) was calculated as (number of positive cells/total cells counted) x 100 .

Caspase-3 mRNA detection. Chloral hydrate (10\%) was intraperitoneally injected for anesthesia. The fur on the heads of the rats was wetted with $75 \%$ ethanol and the head was removed from the neck. The scalp was stripped and a small hole was cut in the skull. After changing gloves, the

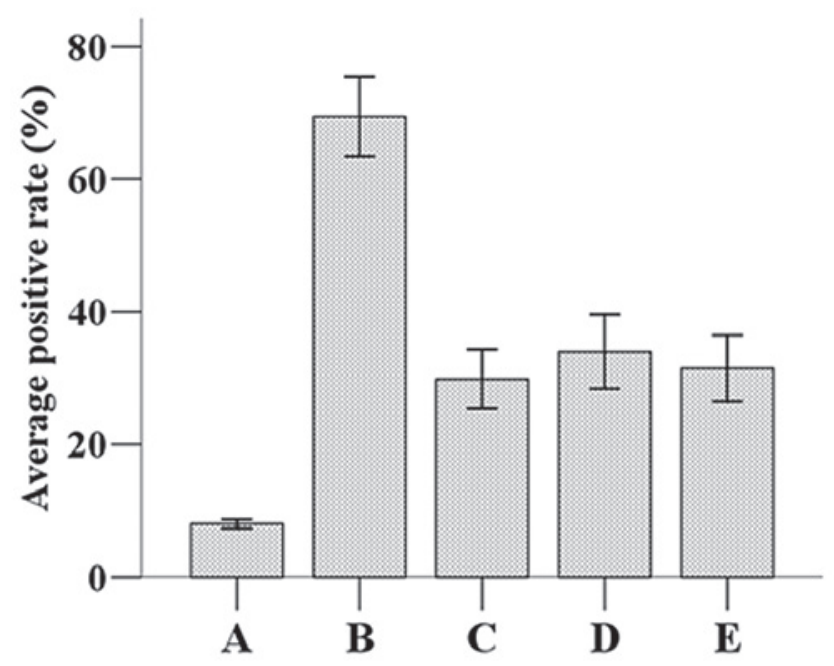

Figure 1. Average positive rate of caspase-3 protein among the groups. A, sham surgery group; B, model group; C, nimodipine group; D, ginkgo leaf tablet group; E, aloe polysaccharide group.

skull was prised open with vessel pincers to expose the brain tissue. Approximately $100 \mathrm{mg}$ of cortex was cut from the parietal lobe of the cerebral ischemic side, using a surgical blade treated with DEPC water: a cut of 7-11 $\mathrm{mm}$ from the coronal plane to frontal pole was made, the fan-shaped ischemic penumbra was separated, and $1 / 3$ of the distance from longitudinal fissure to brain cortex on the lateral brain was removed. The cortex was placed in cold saline to remove the blood and absorb water. The brain tissue was placed in numbered freezing tubes and then into liquid nitrogen and stored in an ultra-low temperature refrigerator at $-80^{\circ} \mathrm{C}$. The reverse transcription-polymerase chain reaction (RT-PCR) was used to detect caspase-3 mRNA expression.

Statistical analysis. SPSS 13.0 statistical software (SPSS, Inc., Chicago, IL, USA) was used for statistical analysis and data were presented as the mean \pm standard deviation. Single-factor analysis of variance was applied to compare the average positive rates of caspase-3 protein and the caspase-3 mRNA/ $\beta$-actin ratio among the groups followed by intergroup, pairwise comparison using the SNK method. The above hypothesis test is two-sided with a test level $\alpha$ of 0.05 . $\mathrm{P}$-values of $<0.05$ were considered to indicate a statistically significant result.

\section{Results}

Caspase- 3 protein expression among the groups. The immunohistochemistry results showed that caspase-3-positive cells exhibited brown particles in the cytoplasm and/or nuclei. Caspase-3 protein expression and the average positive rates among the groups are shown in Fig. 1. The statistical analysis revealed that the average positive rate of caspase-3 protein in the model group $(69.453 \pm 5.747)$ was significantly higher compared with the sham surgery group $(8.039 \pm 0.653$; $\mathrm{P}<0.05)$. The average positive rates of caspase- 3 protein in the nimodipine (29.839 \pm 4.294$)$, Ginkgo biloba $(33.970 \pm 5.328)$ 


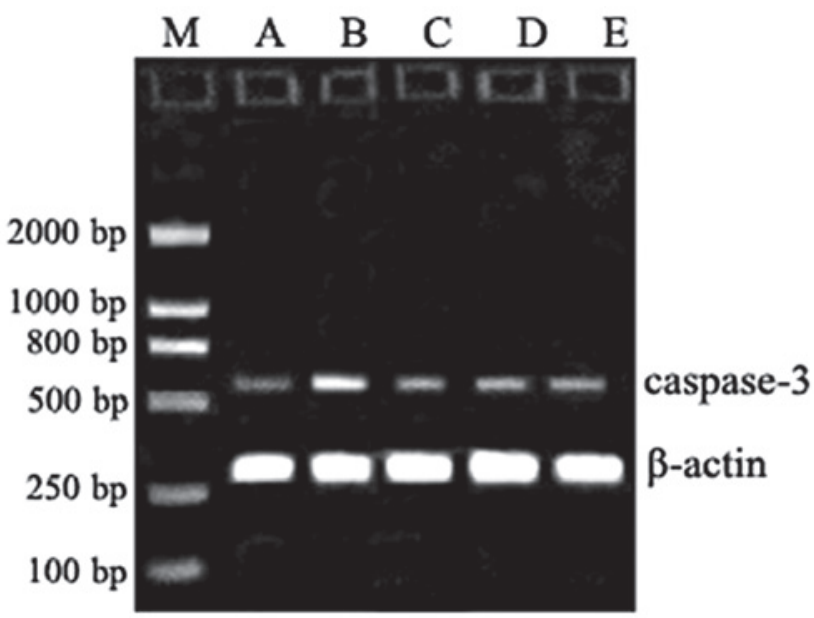

Figure 2. Expression of caspase-3 mRNA among the groups. M, marker; A, sham surgery group; B, model group; C, nimodipine group; D, ginkgo leaf tablet group; E, aloe polysaccharide group.

and the aloe polysaccharide groups $(31.479 \pm 4.763)$ were significantly lower than those in the model group, but higher than those in the sham group $(\mathrm{P}<0.05)$. However, no significant difference was found in the positive rates of caspase-3 protein among the nimodipine, Ginkgo biloba and aloe polysaccharide groups $(\mathrm{P}>0.05)$.

Caspase-3 mRNA expression among the groups. The caspase- 3 mRNA expression and $\mathrm{mRNA} / \beta$-actin values in the groups are shown in Figs. 2 and 3. The statistical analysis revealed that the caspase- $3 / \beta$-actin ratio $(0.286 \pm 0.020)$ was significantly higher compared with the sham group $(0.179 \pm 0.022, \mathrm{P}<0.05)$. The caspase- $3 / \beta$-actin values in the nimodipine $(0.212 \pm 0.012)$, Ginkgo biloba $(0.218 \pm 0.017)$ and the aloe polysaccharide $(0.216 \pm 0.022)$ groups were significantly lower than those in the model group but higher than those in the sham group $(\mathrm{P}<0.05)$. However, no significant difference was observed between the caspase-3/ $\beta$-actin ratio among the nimodipine, Ginkgo biloba and the aloe polysaccharide groups $(\mathrm{P}>0.05)$.

\section{Discussion}

During ischemic brain damage, a series of cell metabolism abnormalities may be induced due to interrupted blood, oxygen and glucose supply. This energy depletion eventually leads to neuronal death or apoptosis (7). Previous studies have revealed that neuronal apoptosis plays a key role in the pathological process of cerebral ischemia-reperfusion injury (7). In the process of apoptosis, proteins that have crucial functions are affected by specific caspase enzymes (8). Caspases belong to a highly conserved cysteine protease family that triggers a cascade of proteolysis. All caspases decompose at substrate-specific aspartic acid residues. Upstream caspases successively activate the downstream apoptosis-promoting executive caspases (9). In the caspase cascade, caspase- 8 , as an initiating protease, activates a series of proteases, including caspase- 9 and -7 , and finally activates caspase- 3 , which is an

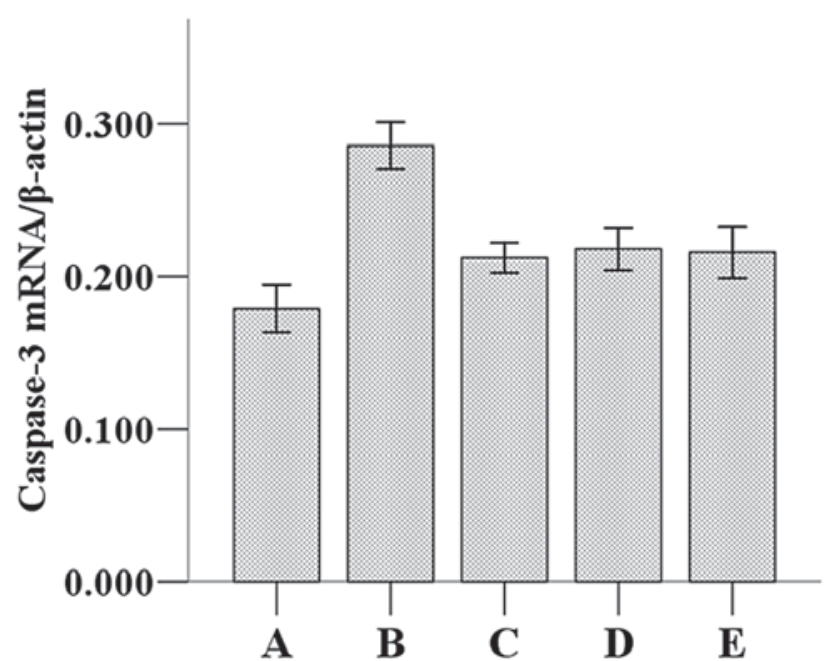

Figure 3. Ratio of caspase-3 mRNA/ $\beta$-actin among the groups. A, sham surgery group; B, model group; C, nimodipine group; D, ginkgo leaf tablet group; E, aloe polysaccharide group.

executor of apoptosis. Caspase-3 is able to incise itself and affect key proteins during apoptosis execution, including the DNA breaks that are typical of apoptosis (10).

In the normal mouse brain, caspase- 3 is expressed at low levels (11). Krupinski et al found that following cerebral ischemic injury, caspase-3 peaks at $12-24 \mathrm{~h}$ in the zone of infarction and the surrounding penumbra in rats (12). According to a study by Harrison et al, compared with the ipsilateral cortex in the control and sham groups and the contralateral cortex of the MCAO rats, the caspase-3 mRNA expression in the ipsilateral cortex (the side with damage) in MCAO rats significantly increased at 6,12 and $24 \mathrm{~h}$, suggesting the involvement of caspase- 3 in infarction (13). The results of the present study reveal that caspase- 3 protein and mRNA expression levels in an ischemia-reperfusion injury model in rats were significantly higher compared with the sham group. This finding indicates that caspase- 3 is involved in the pathological process of cerebral ischemia-reperfusion injury.

While the caspase- 3 protein and mRNA expression levels in rats treated with aloe polysaccharides were lower compared with those in the sham group, they were not significantly different compared with rats treated with nimodipine and Ginkgo biloba. This observation indicates that aloe polysaccharides are able to downregulate caspase-3 expression and have a preventive effect on neuronal apoptosis following ischemic brain injury. The mechanism by which this occurs may be associated with the prevention of the activation of the caspase cascade by regulating cellular proteins or cytokine secretion. It may also be associated with directing the inhibition of caspase- 3 expression and reduced brain neuronal apoptosis. Other relevant anti-ischemic mechanisms require further study and confirmation.

In conclusion, caspase-3 is important during neuronal apoptosis and its downregulation reduces neuronal apoptosis and cerebral ischemia-reperfusion injury. Aloe polysaccharides are able to inhibit caspase- 3 expression, limit apoptosis and provide neuroprotection. 


\section{References}

1. Caplan LR, Searls DE and Hon FK: Cerebrovascular disease. Med Clin North Am 93: 353-369, 2009.

2. Siegel C, Turtzo C and McCullough LD: Sex differences in cerebral ischemia: possible molecular mechanisms. J Neurosci Res 88: 2765-2774, 2010.

3. Broughton BR, Reutens DC and Sobey CG: Apoptotic mechanisms after cerebral ischemia. Stroke 40: 331-339, 2009.

4. Kumar S and Dorstyn L: Analysing caspase activation and caspase activity in apoptotic cells. Methods Mol Biol 559: 3-17, 2009.

5. Li M, Li H, Li C, et al: Alpha fetoprotein is a novel protein-binding partner for caspase-3 and blocks the apoptotic signaling pathway in human hepatoma cells. Int J Cancer 124: 2845-2854, 2009.

6. Longa EZ, Weinstein PR, Carlson S, et al: Reversible middle cerebral artery occlusion without craniectomy in rats. Stroke 20 84-91, 1989.

7. Nakka VP, Gusain A, Mehta SL, et al: Molecular mechanisms of apoptosis in cerebral ischemia: multiple neuroprotective opportunities. Mol Neurobiol 37: 7-38, 2008.
8. Cho BB and Toledo-Pereyra LH: Caspase-independent programmed cell death following ischemic stroke. J Invest Surg 21: 141-147, 2008.

9. Kumar S: Caspase function in programmed cell death. Cell Death Differ 14: 32-43, 2007.

10. Walsh JG, Cullen SP, Sheridan C, et al: Executioner caspase-3 and caspase-7 are functionally distinct proteases. Proc Natl Acad Sci USA 105: 12815-12819, 2007.

11. Shimohama S, Tanino H and Fujimoto S: Differential expression of rat brain caspase family proteins during development and aging. Biochem Biophys Res Commun 289: 1063-1066, 2001.

12. Krupinski J, Lopez E, Marti E, et al: Expression of caspases and their substrates in the rat model of focal cerebral ischemia. Neurobiol Dis 7: 332-342, 2001.

13. Harrison DC, Davis RP, Bond BC, et al: Caspase mRNA expression in a rat model of focal cerebral ischemia. Brain Res Mol Brain Res 89: 133-146, 2001. 